\title{
Host cell entry mediators implicated in the cellular tropism of SARS-CoV-2, the pathophysiology of COVID-19 and the identification of microRNAs that can modulate the expression of these mediators (Review)
}

\author{
PERIKLIS KATOPODIS ${ }^{1,2^{*}}$, HARPAL S. RANDEVA ${ }^{3,4}$, DEMETRIOS A. SPANDIDOS ${ }^{5}$, \\ SAYEH SARAVI ${ }^{1}$, IOANNIS KYROU ${ }^{3,4,6-8^{*}}$ and EMMANOUIL KARTERIS ${ }^{1,2^{*}}$ \\ ${ }^{1}$ Biosciences, College of Health, Medicine and Life Sciences, Brunel University London, Uxbridge UB8 3PH; \\ ${ }^{2}$ Division of Thoracic Surgery, The Royal Brompton and Harefield NHS Foundation Trust, Harefield Hospital, \\ London UB96JH; ${ }^{3}$ Warwickshire Institute for The Study of Diabetes, Endocrinology and Metabolism (WISDEM), \\ University Hospitals Coventry and Warwickshire NHS Trust, Coventry CV2 2DX; ${ }^{4}$ Warwick Medical School, \\ University of Warwick, Coventry CV4 7AL, UK; ${ }^{5}$ Laboratory of Clinical Virology, Medical School, University of Crete, \\ 71409 Heraklion, Greece; ${ }^{6}$ Centre for Sport, Exercise and Life Sciences, Research Institute for Health and Wellbeing, \\ Coventry University, Coventry CV1 5FB; ${ }^{7}$ Aston Medical School, College of Health and Life Sciences, \\ Aston University, Birmingham B4 7ET, UK; ${ }^{8}$ School of Food and Nutritional Sciences, \\ Department of Food Science and Human Nutrition, Agricultural University of Athens, 11855 Athens, Greece
}

Received November 16, 2021; Accepted December 15, 2021

DOI: $10.3892 /$ ijmm.2021.5075

\begin{abstract}
The pathophysiology of coronavirus disease 2019 (COVID-19) is mainly dependent on the underlying mechanisms that mediate the entry of severe acute respiratory syndrome coronavirus 2 (SARS-CoV-2) into the host cells of the various human tissues/organs. Recent studies have indicated a higher order of complexity of the mechanisms of
\end{abstract}

Correspondence to: Dr Emmanouil Karteris, Biosciences, College of Health, Medicine and Life Sciences, Brunel University London, Kingston Lane, Uxbridge UB8 3PH, UK

E-mail: emmanouil.karteris@brunel.ac.uk

Dr Ioannis Kyrou, Warwickshire Institute for The Study of Diabetes, Endocrinology and Metabolism (WISDEM), University Hospitals Coventry and Warwickshire NHS Trust, Clifford Bridge Road, Coventry CV2 2DX, UK

E-mail:kyrouj@gmail.com

*Contributed equally

Abbreviations: COVID-19, coronavirus disease 2019; SARS-CoV2, severe acute respiratory syndrome coronavirus 2; ACE2, angiotensin converting enzyme 2; NPR1, neuropilin-1; TMPRSS2 and 4 , transmembrane protease serine 2 and 4; ADAM17, ADAM metallopeptidase domain 17 (also termed tumour necrosis factor- $\alpha$ convertase); KIM1, kidney injury molecule-1; TLR4, Toll-like receptor 4; GRP78, glucose-regulated protein 78; miRNAs, microRNAs

Key words: COVID-19, SARS-CoV2, ACE2, NPR1, TMPRSS2, TMPRSS4, ADAM17, TLR4, GRP78, miRNAs infectivity, given that there is a wide-repertoire of possible cell entry mediators that appear to co-localise in a cell- and tissue-specific manner. The present study provides an overview of the 'canonical' SARS-CoV-2 mediators, namely angiotensin converting enzyme 2 , transmembrane protease serine 2 and 4, and neuropilin-1, expanding on the involvement of novel candidates, including glucose-regulated protein 78 , basigin, kidney injury molecule-1, metabotropic glutamate receptor subtype 2, ADAM metallopeptidase domain 17 (also termed tumour necrosis factor- $\alpha$ convertase) and Toll-like receptor 4 . Furthermore, emerging data indicate that changes in microRNA (miRNA/miR) expression levels in patients with COVID-19 are suggestive of further complexity in the regulation of these viral mediators. An in silico analysis revealed 160 candidate miRNAs with potential strong binding capacity in the aforementioned genes. Future studies should concentrate on elucidating the association between the cellular tropism of the SARS-CoV-2 cell entry mediators and the mechanisms through which they might affect the clinical outcome. Finally, the clinical utility as a biomarker or therapeutic target of miRNAs in the context of COVID-19 warrants further investigation.

\section{Contents}

1. Introduction

2. Angiotensin converting enzyme 2, and transmembrane protease serine 2 and 4

3. Neuropilin-1

4. Emerging SARS-CoV-2 cell entry mediators 
5. Identification of miRNAs that can target and regulate the gene expression of SARS-CoV-2 cell entry mediators

6. Conclusion

\section{Introduction}

Severe acute respiratory syndrome coronavirus 2 (SARS-CoV-2) is an enveloped RNA virus that is transmitted mainly via air droplets, and can cause a viral infectious disease in humans, namely coronavirus disease 2019 (COVID-19), which was first identified towards the end of 2019 (1). COVID-19 manifestations range from mild (asymptomatic or mild respiratory tract infection in the majority of reported cases) to severe and the disease can be fatal in high-risk individuals, with a spectrum of respiratory and/or extra-pulmonary manifestations that may require hospitalization and potentially mechanical ventilation and intensive care unit support (1). Of note, SARS-CoV-2 is highly virulent, has a tendency to mutate and exhibits a cellular tropism within the human body that appears to be associated with the expression of various host cell entry mediators, such as the angiotensin converting enzyme 2 (ACE2) (2-4). The present review article presents a concise overview of the key host cell entry mediators which have been identified to play a role in the infection of human cells by SARS-CoV-2, summarizing the evidence on the mechanisms through which ACE2 and additional host receptors/proteins appear to be implicated in the cellular tropism of SARS-CoV-2 and the pathophysiology of COVID-19. Moreover, the present review article aims to provide insight into the mechanisms through which these cell entry mediators may be regulated by certain microRNAs (miRNAs/miRs).

\section{Angiotensin converting enzyme 2, and transmembrane protease serine 2 and 4}

Both SARS-CoV and SARS-CoV-2 have been reported to use ACE2 as the key receptor for their host cell entry (4-6). Indeed, an initial step in these viral infections is the binding of this cellular receptor with the viral spike $\mathrm{S}$ glycoprotein (S protein), which consists of the S1 and S2 subunits (Fig. 1). The $\mathrm{S} 1$ subunit contains a $\mathrm{C}$-terminal receptor-binding domain that recognizes and binds to the specific host receptor (e.g., ACE2), whilst the S2 subdomain is responsible for the fusion of the viral membrane with the host cell membrane via the fusion peptide. Following this binding to ACE2, a protease cleavage of the $\mathrm{S}$ protein follows. This process is catalysed by transmembrane protease serine (TMPRSS)2, and other host proteases, such as cathepsin L and furin at the S1-S2 site, which activate the entry of SARS-CoV-2 (5,7-11).

Based on consistent data, ACE2 is now well-established as the key mediator that facilitates the entry of SARS-CoV-2 into human host cells $(6,12,13)$. Notably, ACE2 is highly expressed in the kidneys, small intestine, prostate and lung alveolar epithelial type II cells, which explains the documented tropism of this novel coronavirus and the related pulmonary and extrapulmonary symptoms that a SARS-CoV-2 infection may cause (14). The analysis of autopsy tissue from fatal COVID-19 cases has most frequently detected SARS-CoV-2 RNA in the airway epithelium, whilst SARS-CoV-2 RNA has also been found to be highly co-localised in cells expressing
TMPRSS2 (15-18). Of note, it has been suggested that DNA polymorphisms for ACE2 and/or TMPRSS2 may be associated with the genetic susceptibility of different populations to severe COVID-19 infection (9). Along with TMPRSS2, TMPRSS4 has also been shown to be involved in the infectivity of SARS-CoV-2. In human small intestinal enterocytes, the presence of both enzymes drives the cleavage of the spike $\mathrm{S}$ glycoprotein of SARS-CoV-2 and enhances subsequent membrane fusion (19). Moreover, TMPRSS2 and TMPRSS4, along with ACE2, are abundantly co-expressed in the small intestine and colon (20). Subsequently, the authors have previously demonstrated that TMPRSS4 is overexpressed in 11 types of cancer in the colon mucosa and in all the brain regions that are associated with the sense of taste and smell (21).

\section{Neuropilin-1}

It is noteworthy that the relatively low/moderate expression of ACE2 in the human respiratory system has triggered further research focusing on the discovery of potential additional cell proteins/receptors that may mediate the SARS-CoV-2 infectivity and contribute to the selective tissue/organ tropism of this new coronavirus $(22,23)$. In this context, two elegant studies by Daly et al (24) and Cantuti-Castelvetri (25) et al have demonstrated that neuropilin (NRP)1 constitutes an additional cellular mediator implicated in the entry of SARS-CoV-2 into human cells. Indeed, based on the experimental data of both these studies, it is evident that, not only ACE2, but also NRP1 can act as a host cell mediator that enhances the infectivity of SARS-CoV-2 and contributes to its documented tissue/organ tropism.

Overall, NRPs are classed as non-tyrosine kinase, single-transmembrane glycoproteins which are greatly conserved in vertebrates and constitute co-receptors for a number of molecules, such as semaphorins and vascular endothelial growth factors (VEGFs), playing a key role in numerous physiological processes (e.g., neuronal development and axon control, immune function angiogenesis, cell proliferation and vascular permeability) (23-26).

Of note, the aforementioned studies by Daly et al (24) and Cantuti-Castelvetri et al (25) suggest that, unlike SARS-CoV $\mathrm{S}$ proteins, SARS-CoV-2 S proteins have a polybasic sequence motif (Arg-Arg-Ala-Arg) at the S1-S2 junction/boundary that conforms to the 'C-end rule' (CendR motif) [R-XX-R; where $\mathrm{R}$ denotes arginine (Arg), can be replaced by lysine (Lys)] and serves as a cleavage site for the convertase furin (Fig. 1) (24-26). Thus, SARS-CoV-2 can enter host cells more easily with the aid of NRP1, boosting its infectivity and promoting its tropism $(24,25)$. Indeed, with the use of human cell lines, it has been shown that NRP1 enhances infection by a clinical SARS-CoV-2 isolate and lentiviral particles pseudotyped with the $S$ protein of SARS-CoV-2 by binding to this CendR motif of the furin-cleaved SARS-CoV-2 S1 protein. This NRP1-mediated increase in SARS-CoV-2 infectivity appears to be due to increased viral entry into host cells, rather than increased viral binding to the cell membrane, whilst it is amplified when ACE2 and TMPRSS2 are present. On the other hand, the rate of NRP1-enhanced SARS-CoV-2 infection in human cell cultures has been shown to be suppressed by using a monoclonal blocking antibody against the extracellular NRP1 b1b2 space, or a specific NRP1 mutant which ties the CendR-binding b1 domain $(24,26,27)$. In addition, 


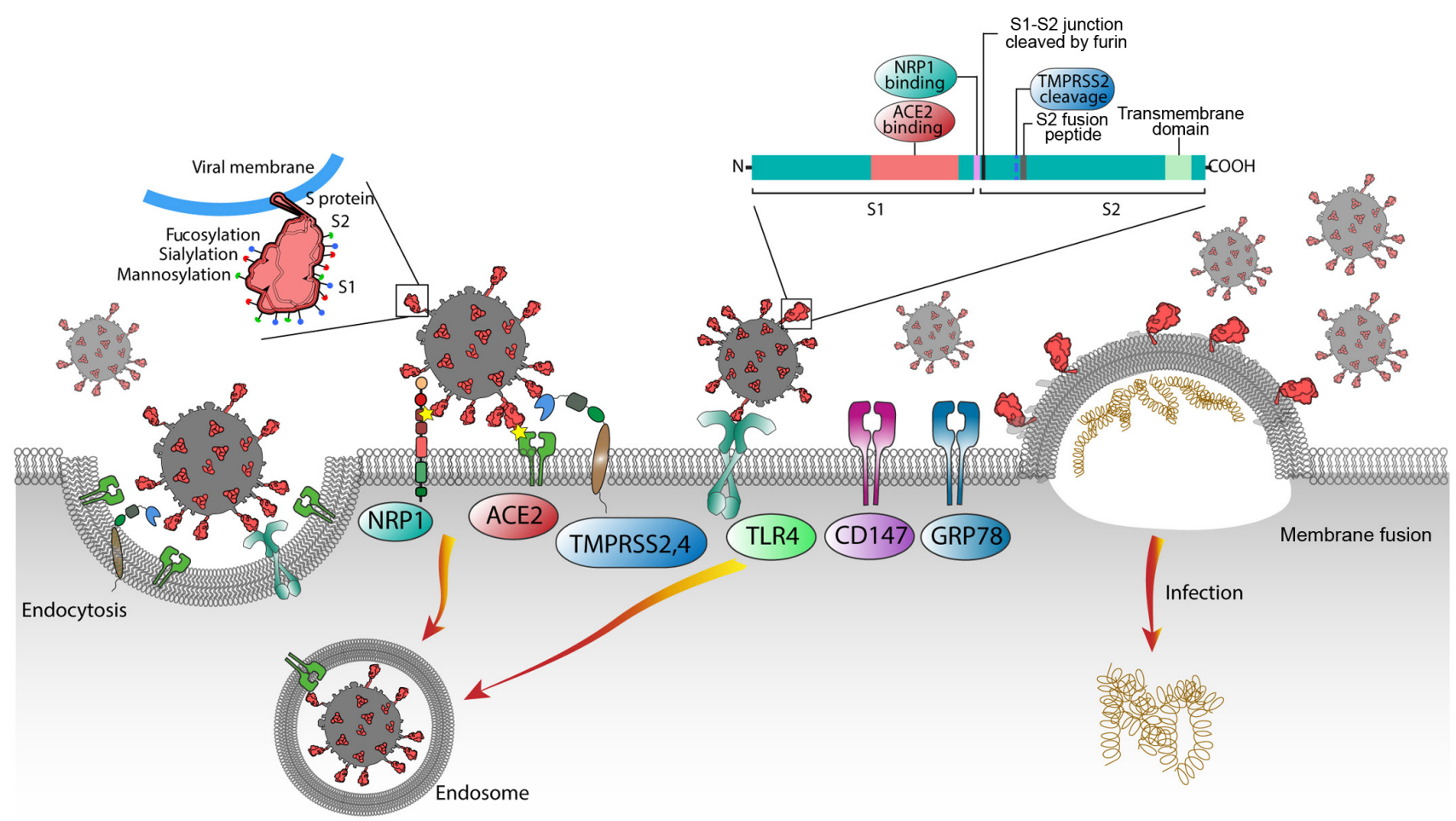

Figure 1. Schematic diagram of the known pathways facilitating the entry of SARS-CoV-2 into host cells. The SARS-CoV-2 spike (S) glycoproteins are instrumental for this process; S1 (being the cell connecting head of the molecule) binds to the ACE2 receptor, while S2 mediates the viral-cell membrane fusion by being exposed to TMPRSS2 and TMPRSS4 or other host cell proteases, such as furin, that is cleaved at the S1-S2 junction and allows the binding of S1 to NRP1. Additional molecules, including TLR4, CD147 and GRP78, have also been proposed as potential SARS-CoV-2 cell entry mediators. SARS-CoV2, severe acute respiratory syndrome coronavirus 2; ACE2, angiotensin converting enzyme 2; NPR1, neuropilin-1; TMPRSS2 and 4, transmembrane protease serine 2 and 4; TLR4, Toll-like receptor 4; GRP78, glucose-regulated protein 78; CD147, basigin (BSG).

as previously demonstrated, mutated variants of SARS-CoV-2 with a modified furin cleavage site of the $S$ protein (erased polybasic cleavage site or impervious to furin-intervened cleavage) were not subject to NRP1-mediated infectivity, and similarly transformations in the NRP1 b1 domain likewise restrained the NRP1-S1 interaction $(24,26,27)$.

Notably, the upregulation of NRP1 gene expression has also been demonstrated in lung tissue samples and infected olfactory epithelial cells from patients with COVID-19; further co-staining revealed the infection of cells that were positive for oligodendrocyte transcription factor 2 , which is primarily expressed by olfactory neuronal progenitors (27).

Given these data on NRP1 and since extra-pulmonary symptoms (e.g., gastrointestinal and neurological symptoms) are frequently identified in relation to COVID-19, extensive additional research is still required to identify the association between SARS-CoV-2 tropism and the mechanisms through which host cell infection mediators, such as NRP1, are dispersed across the various tissues/organs of the human body. This may further aid in the development of novel antiviral drugs against COVID-19, which for example could be targeting NRP1 and its role in promoting the entry of SARS-CoV-2 into host cells.

\section{Emerging SARS-CoV-2 cell entry mediators}

Following the initial findings regarding the role of host cell receptors/mediators, such as ACE2 and NRP1, data are also emerging for a number of additional cellular factors which may also facilitate or inhibit the infectivity of SARS-CoV-2, including glucose-regulated protein 78 (GRP78), basigin (BSG; CD147), kidney injury molecule-1 (KIM1), heparan sulfate (HS), ADAM metallopeptidase domain 17 [also termed tumour necrosis factor- $\alpha$ convertase (ADAM17)], surfactant protein D (SP-D), metabotropic glutamate receptor subtype 2 (mGluR2) and Toll-like receptor 4 (TLR4).

In this context, heat shock protein A5 (HSPA5), also termed GRP78, has been reported to be implicated in a possible route for SARS-CoV-2 cell attachment and entry. Indeed, it has been proposed that the recognition site for GRP78 is found in SARS-CoV-2 (28), and that the binding is more favourable between regions III (C391-C525) and IV (C480-C488) of the SARS-CoV-2 protein spike model and GRP78 (29). Moreover, CD147 (also known as EMMPRIN or BSG; a transmembrane glycoprotein that belongs to the large immunoglobulin superfamily) has also been proposed as another molecule that can facilitate the entry of SARS-CoV-2 into host cells by endocytosis. This is supported by the findings of an in vitro study in which SARS-CoV-2 amplification was inhibited when CD147 was blocked in Vero E6 and BEAS-2B cell lines (30). However, based on the findings of a later study, another group argued that there was no evidence for a direct interaction between the viral spike protein and this particular molecule (31). Such contradictory findings highlight the current need for further research into the entire spectrum of potential host-pathogen interactions for SARS-CoV-2.

Furthermore, given that kidney-related complications are common in patients with COVID-19 (32), the involvement of KIM1 has been recently investigated. Accordingly, through a 
series of experiments, it was previously demonstrated that KIM1 is capable of interacting with the receptor-binding domain of this new coronavirus, subsequently enabling its attachment to the plasma membrane (33). These findings suggest that this molecule may mediate and exacerbate SARS-CoV-2 infection of the kidneys (33). Moreover, KIM1 may function as a biomarker of acute kidney injury, which has been associated with a poor prognosis of patients with COVID-19 (34).

mGluR2 (GRM2), a G protein-coupled receptor which inhibits adenylyl cyclase and regulates the mechanisms through which glutamate can control cell excitability, is another potential cell entry receptor for SARS-CoV-2. Indeed, recent experiments have demonstrated that mGluR2 interacts directly with the $S$ protein of SARS-CoV-2, whilst the knockdown of mGluR2, even though it does not affect binding to the plasma membrane, reduces the clathrin-mediated endocytosis of this virus (35). Moreover, in vivo studies corroborated these findings, where viral infectivity in lungs of mice was significantly reduced, when mGluR2 was knocked out (35). Of note, angiotensin II receptor type 2 is another $\mathrm{G}$ protein-coupled receptor, which, based on an in silico simulation data has revealed an interaction with the $\mathrm{S}$ protein of SARS-CoV-2 (36). However, further in vitro and/or in vivo studies are required to validate these initial modelling data.

Apart from direct cell entry mediators of SARS-CoV-2 into host cells, there have been molecules which appear to function as co-factors in this process, such as HS and ADAM17. Of note, HS is a co-factor for ACE2-mediated viral entry and a potential therapeutic target (e.g., using mitoxantrone) (37). On the other hand, ADAM17 has been shown to regulate the shedding of the ACE2 ectodomain (38). This is in line with its role in releasing ectodomains of a wide repertoire of cytokines, enzymes or other molecules $(38,39)$. However, due to a potential redundancy in viral cell entry, it has been demonstrated that TMPRSS2 can compete with ADAM17 for ACE2 processing (40). Furthermore, SP-D is an innate immune molecule with a main role in clearing pathogens and apoptotic/necrotic cells from pulmonary and extra-pulmonary mucosal sites (41). Given that human SP-D can recognise the spike glycoprotein of SARS-CoV, a recent study demonstrated that the treatment of 293T cells overexpressing ACE2 with a recombinant fragment of human SP-D (rfhSP-D) inhibited the interaction of the S1 spike proteins with the cell membrane, raising the possibility of an alternative therapeutic target (42). Indeed, in another study, when clinical samples were used, treatment with rfhSP-D inhibited viral replication, and appeared to be more efficient than the antiviral drug remdesivir, when Vero cells were treated in vitro (43). Currently, a Phase 1 clinical trial (ClinicalTrials.gov identifier: NCT04659122) is ongoing, entitled: 'Phase 1b Open-label, Single Arm, Cohort Dose Escalation Study Evaluating the Safety, Tolerability, and Feasibility of Intervention With AT-100 (rhSP-D) in Intubated Patients Receiving Invasive Mechanical Ventilation With Severe COVID-19 Infection'. Finally, in a previous observational study on 39 patients with COVID-19, SP-D levels were found to be significantly higher in severe compared to mild cases, suggesting the possibility of its use as a biomarker of severity as well (44).

Finally, another innate immune molecule that has gained increasing interest in the context of COVID-19 is TLR4, which represents a key cell surface receptor that induces the secretion of pro-inflammatory cytokines and interferons to fight infection (45). Indeed, there is accumulating evidence to indicate that SARS-CoV-2 can bind to TLR4, whilst treatment with resatorvid (a TLR4 specific inhibitor) has been shown to abolish the secretion of IL1B by SARS-CoV-2 in THP- 1 cells $(46,47)$.

Overall, identifying the underlying mechanisms that facilitate the entry of SARS-CoV-2 into host cells (Fig. 1) is currently the focus of intensive research which is expected to provide further valuable insight into the pathophysiology of COVID-19, identifying the human tissues/organs that are more vulnerable to a SARS-CoV-2 infection. Ultimately, this may also aid in the development of more precise treatments against COVID-19 sequelae. A list of the 'canonical' and potential cell entry mediators is provided in Table I.

\section{Identification of miRNAs that can target and regulate the gene expression of SARS-CoV-2 cell entry mediators}

miRNAs are single-strand, endogenous, non-coding, short RNAs of 22-25 nucleotides in length, which can regulate gene expression post-transcriptionally and subsequently alter signalling pathways (53). A series of review articles have elegantly summarised the functions of miRNAs in health and disease (54-57). Of note, miRNAs regulate the expression of key inflammatory cytokines involved in the massive recruitment of immune cells to the lungs, such as IL6 and TNFa. Several studies have demonstrated that changes in the expression of miRNAs in patients with COVID-19 may be a predictor of tissue damage and lung inflammation, whilst miRNAs can also be potent diagnostic biomarkers for COVID-19 (58-60).

Several miRNAs have been either predicted or shown to target SARS-CoV-2 RNAs (61). Indeed, a number of miRNAs have been found to target the RNA $S$ glycoprotein sequence of SARS-CoV-2, which interacts with ACE2 for viral entry into host cells, including hsa-miR-4661-3p (62), hsa-miR-510-3p, hsa-miR-624-5p, hsa-miR-497-5p (63), hsa-miR-622, hsa-miR-761, miR-A3r, hsa-miR-15b-5p, miR-A2r, hsa-miR-196a-5p (64), and miR-338-3p, miR-4661-3p, miR-4761-5p, hsa-miR-4464, hsa-miR-1234-3p, hsa-miR-7107-5p and hsa-miR-885-5p, which have been shown to bind to the receptor binding domain of the $\mathrm{S}$ gene (62).

As such, a number of studies have identified host cell miRNAs that target different SARS-CoV-2 proteins. Considering the aforementioned significance of ACE2 and TMPRSS2 in SARS-CoV-2 infection, in a previous study, a TargetScan analysis revealed several miRNAs that could directly target these two receptors, including hsa-mi-200b-3p, hsa-miR-200c-3p and hsa-miR-429 for ACE2, and hsa-let7a-5p, hsa-let7b-5p, hsa-let7c-5p, hsa-let7d-5p, hsa-let7e-5p, hsa-let7f-5p, hsa-let7g-5p, hsa-let7i-5p, hsa-miR-98-5p, has-miR-4458 and hsa-miR-4500 for TMPRSS2; thus, these miRNAs may be utilised as potent therapeutic molecules to regulate key proteins that are required for viral entry to the host airway/lung epithelial cells (59). Moreover, TMPRSS2, which activates the spike protein of SARS-CoV-2 to promote viral infection, has been predicted to be regulated by MR147-3p in the gut (62).

Overall, several cell host miRNAs that target the ACE2 and TMPRSS2 genes can prevent attachment and the entry of SARS-CoV-2 into different tissues. For example, hsa-miR-98-5p has been shown to directly target the 3'-UTR of TMPRSS2, both in human lung microvascular endothelial cells (HMVEC-L) and human umbilical vein 
Table I. Genes related to SARS-CoV-2 entry into host cells.

\begin{tabular}{|c|c|c|c|}
\hline Cell entry mediators & Abbreviation & Proposed action & (Refs.) \\
\hline Angiotensin converting enzyme 2 & ACE2 & Canonical receptor for the S glycoprotein (S1 domain) & $(4,48,49)$ \\
\hline Transmembrane protease, serine 2 and & $\begin{array}{l}\text { TMPRSS2 } \\
\text { TMPR SS4 }\end{array}$ & Mediate fusion of viral and host cell membranes after & $(7,19,50,51)$ \\
\hline $\begin{array}{l}\text { serine } 4 \\
\text { Neuropilin-1 }\end{array}$ & $\begin{array}{l}\text { TMPRSS4 } \\
\text { NRP1 }\end{array}$ & $\begin{array}{l}\text { proteolytic cleavage at the S1/S2 region } \\
\text { Additional SARS-CoV-2 host cell receptor, increasing } \\
\text { its infectivity and contributing to its tropism }\end{array}$ & $(20,23-25,48)$ \\
\hline Toll-like receptor 4 & TLR4 & Potential alternative receptor for SARS-CoV-2 & $(46,47,52)$ \\
\hline Kidney injury molecule-1 & KIM1 & $\begin{array}{l}\text { Potential interaction with SARS-CoV-2 } \\
\text { receptor-binding domain }\end{array}$ & $(33,34)$ \\
\hline Basigin & BSG/CD147 & $\begin{array}{l}\text { Potential facilitator of the entry of SARS-CoV- } 2 \text { into } \\
\text { host cells by endocytosis }\end{array}$ & $(30,31)$ \\
\hline $\begin{array}{l}\text { Heat shock protein } \mathrm{A} 5 \text { or glucose } \\
\text { regulating protein } 78\end{array}$ & HSPA5/GRP78 & $\begin{array}{l}\text { Possible route for SARS-CoV-2 cell attachment } \\
\text { and entry }\end{array}$ & $(28,29)$ \\
\hline $\begin{array}{l}\text { ADAM metallopeptidase domain } 17 \\
\text { or TNF- } \alpha \text { convertase enzyme }\end{array}$ & ADAM17 & Regulates shedding of the ACE 2 ectodomain & $(10,38,39)$ \\
\hline
\end{tabular}

SARS-CoV2, severe acute respiratory syndrome coronavirus 2 .

Table II. List of miRNAs that indicate strong binding potential against transmembrane serine protease 4.

\begin{tabular}{lcll}
\hline Target rank & Target score & \multicolumn{1}{c}{ miRNA name } & \multicolumn{1}{c}{ miRNA sequence } \\
\hline 1 & 88 & hsa-miR-6721-5p & $5^{\prime}$-ugggcaggggcuuauuguaggag-3' \\
2 & 85 & hsa-miR-3671 & $5^{\prime}$-aucaaauaaggacuagucugca-3' \\
3 & 82 & hsa-miR-496 & $5^{\prime}$-ugaguauuacauggccaaucuc-3' \\
4 & 81 & hsa-miR-551b-3p & $5^{\prime}$-gcgacccauacuugguuucag-3' \\
5 & 81 & hsa-miR-551a & $5^{\prime}$-gcgacccacucuugguuucca-3' \\
\hline
\end{tabular}

endothelial cells (65). Furthermore, the hsa-let-7e/hsa-mir-125a and hsa-mir-141/hsa-miR-200 miRNA families inhibit ACE2 and TMPRSS2 expression (66). It has also been shown that miR-200c is essential for SARS-CoV-2 binding to the ACE2 receptor and entry into cardiomyocytes (67), while miR-98-5p can inhibit TMPRSS2 expression in human endothelial cells (65). In a previous study, bioinformatics analysis revealed that lysine-specific demethylase 5B can regulate ACE2 and TMPRSS2 via the transcriptional repression of let-7e/miR-125a and $\mathrm{miR}-141 / \mathrm{miR}-200 \mathrm{miRNAs}$; thus, these miRNAs are crucial for the function of ACE2 and TMPRSS2 (66).

Notably, a recent study provided novel evidence of an interplay between host miRNAs and SARS-CoV-2, as well as the mechanisms through which these interactions can drive pathogenesis by dysregulating certain immune pathways (68). A recent mini-review, provided an excellent overview of the role of COVID-19-associated miRNAs as potential therapeutic targets and biomarkers (69). Currently, there are a number of clinical trials/studies investigating miRNAs in the context of COVID-19. For example, in an observational study on the expression of cytokines, transcriptome and miRNAs in COVID-19 patients is currently recruiting (ClinicalTrials. gov identifier: NCT04583566). Similarly, 'The Effect of miRNA and Epigenetic Modifications on Prognosis in Covid-19 Infection' will also be investigated (ClinicalTrials. gov Identifier: NCT04411563), whilst another study aims to assess the mechanisms through which miRNA levels relate to viral infection (ClinicalTrials.gov Identifier: NCT04346160). In terms of therapeutics, a phase 2 trial is currently enrolling by invitation, with miRNA-containing exosomes as an aerosol inhaler (ClinicalTrials.gov Identifier: NCT04602442).

Of note, the targeting of PARP-1 by miRNAs has been suggested to hold therapeutic value for COVID-19-related pathologies (70). In a recent in silico study, a number of hypothalamic miRNAs that can bind and regulate ACE2 and TMPRSS2 were identified (71). The authors of that study concluded that these miRNAs may be used in the search for novel therapies for the neurological symptoms in patients with COVID-19. The present study expanded on these initial observations by identifying potential miRNA modulators of all remaining SARS-CoV-2 cell entry mediators, using miRDB, an online database for miRNA target prediction and functional annotations (mirdb.org) (72,73). For the present study, the target prediction score was set $>80$ for higher confidence in the interactions. As such, Table II provides a list of five potential candidate miRNAs against TMPRRS4; Table III provides a list of 69 miRNAs for NRP1; Table IV indicates 36 potential miRNA interactions with ADAM17; Table V presents five interactions with KIM1; Table VI presents a list of 27 interactions with GRP78 (HSPA5); Table VII presents 
Table III. List of miRNAs that indicate strong binding potential against neuropilin-1.

\begin{tabular}{|c|c|c|c|}
\hline Target rank & Target score & miRNA name & miRNA sequence \\
\hline 1 & 98 & hsa-miR-3646 & 5'-aaaaugaaaugagcccagccca-3' \\
\hline 2 & 97 & hsa-miR-7977 & 5'-uucccagccaacgcacca-3' \\
\hline 3 & 96 & hsa-miR-6844 & 5'-uucuuuguuuuuaauucacag-3' \\
\hline 4 & 95 & hsa-miR-124-3p & 5'-uaaggcacgcggugaaugccaa-3' \\
\hline 5 & 95 & hsa-miR-3133 & 5'-uaaagaacucuuaaaacccaau-3' \\
\hline 6 & 95 & hsa-miR-5094 & 5'-aaucagugaaugccuugaaccu-3' \\
\hline 7 & 95 & hsa-miR-506-3p & 5'-uaaggcacccuucugaguaga-3' \\
\hline 8 & 95 & hsa-miR-153-5p & 5'-ucauuuuugugauguugcagcu-3' \\
\hline 9 & 94 & hsa-miR-4755-5p & 5'-uuucccuucagagccuggcuuu-3' \\
\hline 10 & 94 & hsa-miR-148a-3p & 5'-ucagugcacuacagaacuuugu-3' \\
\hline 11 & 94 & hsa-miR-152-3p & 5'-ucagugcaugacagaacuugg-3' \\
\hline 12 & 94 & hsa-miR-148b-3p & 5'-ucagugcaucacagaacuuugu-3' \\
\hline 13 & 94 & hsa-miR-3148 & 5'-uggaaaaaacuggugugugcuu-3' \\
\hline 14 & 94 & hsa-miR-5006-3p & 5'-uuucccuuuccauccuggcag-3' \\
\hline 15 & 93 & hsa-miR-1285-3p & 5'-ucugggcaacaaagugagaccu-3' \\
\hline 16 & 93 & hsa-miR-548an & 5'-aaaaggcauugugguuuuug-3' \\
\hline 17 & 93 & hsa-miR-3686 & 5'-aucuguaagagaaaguaaauga-3' \\
\hline 18 & 93 & hsa-miR-651-3p & 5'-aaaggaaaguguauccuaaaag-3' \\
\hline 19 & 93 & hsa-miR-5189-5p & 5'-ucugggcacaggcggauggacagg-3' \\
\hline 20 & 92 & hsa-let-7a-3p & 5'-cuauacaaucuacugucuuuc-3' \\
\hline 21 & 92 & hsa-miR-98-3p & 5'-cuauacaacuuacuacuuuccc-3' \\
\hline 22 & 92 & hsa-miR-6860 & 5'-acugggcagggcuguggugagu-3' \\
\hline 23 & 92 & hsa-miR-1-3p & 5'-uggaauguaaagaaguauguau-3' \\
\hline 24 & 92 & hsa-miR-612 & 5'-gcugggcagggcuucugagcuccuu-3' \\
\hline 25 & 92 & hsa-miR-3187-5p & 5'-ccugggcagcguguggcugaagg-3' \\
\hline 26 & 92 & hsa-let-7b-3p & 5'-cuauacaaccuacugccuuccc-3' \\
\hline 27 & 92 & hsa-miR-206 & 5'-uggaauguaaggaagugugugg-3' \\
\hline 28 & 92 & hsa-miR-5004-5p & 5'-ugaggacagggcaaauucacga-3' \\
\hline 29 & 92 & hsa-let-7f-1-3p & 5'-cuauacaaucuauugccuuccc-3' \\
\hline 30 & 91 & hsa-miR-510-3p & 5'-auugaaaccucuaagagugga-3' \\
\hline 31 & 90 & hsa-miR-320d & 5'-aaaagcuggguugagagga-3' \\
\hline 32 & 90 & hsa-miR-613 & 5'-aggaauguuccuucuuugcc-3' \\
\hline 33 & 90 & hsa-miR-4429 & 5'-aaaagcugggcugagaggcg-3' \\
\hline 34 & 90 & hsa-miR-320c & 5'-aaaagcuggguugagagggu-3' \\
\hline 35 & 90 & hsa-miR-6806-3p & 5'-ugaagcucugacauuccugcag-3' \\
\hline 36 & 90 & hsa-miR-3928-5p & 5'-ugaagcucuaagguuccgccugc-3' \\
\hline 37 & 90 & hsa-miR-320a-3p & 5'-aaaagcuggguugagagggcga-3' \\
\hline 38 & 90 & hsa-miR-338-3p & 5'-uccagcaucagugauuuuguug-3' \\
\hline 39 & 90 & hsa-miR-9-5p & 5'-ucuuugguuaucuagcuguauga-3' \\
\hline 40 & 90 & hsa-miR-1322 & 5'-gaugaugcugcugaugcug-3' \\
\hline 41 & 90 & hsa-miR-320b & 5'-aaaagcuggguugagagggcaa-3' \\
\hline 42 & 89 & hsa-miR-150-3p & 5'-cugguacaggccugggggacag-3' \\
\hline 43 & 89 & hsa-miR-4261 & 5'-aggaaacagggaccca-3' \\
\hline 44 & 88 & hsa-miR-6733-3p & 5'-ucagugucuggauuuccuag-3' \\
\hline 45 & 88 & hsa-miR-5701 & 5'-uuauugucacguucugauu-3' \\
\hline 46 & 87 & hsa-miR-3920 & 5'-acugauuaucuuaacucucuga-3' \\
\hline 47 & 87 & hsa-miR-147b-5p & 5'-uggaaacauuucugcacaaacu-3' \\
\hline 48 & 87 & hsa-miR-4724-5p & 5'-aacugaaccaggagugagcuucg-3' \\
\hline 49 & 87 & hsa-miR-4251 & 5'-ccugagaaaagggccaa-3' \\
\hline 50 & 86 & hsa-miR-137-3p & 5'-uuauugcuuaagaauacgcguag-3' \\
\hline 51 & 86 & hsa-miR-4789-5p & 5'-guauacaccugauauguguaug-3' \\
\hline 52 & 86 & hsa-miR-570-3p & 5'-cgaaaacagcaauuaccuuugc-3' \\
\hline
\end{tabular}


Table III. Continued.

\begin{tabular}{|c|c|c|c|}
\hline Target rank & Target score & miRNA name & miRNA sequence \\
\hline 53 & 85 & hsa-miR-4801 & 5'-uacacaagaaaaccaaggcuca-3' \\
\hline 54 & 84 & hsa-miR-24-3p & 5'-uggcucaguucagcaggaacag-3' \\
\hline 55 & 84 & hsa-miR-587 & 5'-uuuccauaggugaugagucac-3' \\
\hline 56 & 83 & hsa-miR-4324 & 5'-cccugagacccuaaccuuaa-3' \\
\hline 57 & 83 & hsa-miR-181a-2-3p & 5'-accacugaccguugacuguacc-3' \\
\hline 58 & 82 & hsa-miR-186-5p & 5'-caaagaauucuccuuuugggcu-3' \\
\hline 59 & 82 & hsa-let-7f-2-3p & 5'-cuauacagucuacugucuuucc-3' \\
\hline 60 & 82 & hsa-miR-6843-3p & 5'-auggucuccuguucucugcag-3' \\
\hline 61 & 82 & hsa-miR-1185-1-3p & 5'-auauacagggggagacucuuau-3' \\
\hline 62 & 82 & hsa-miR-6848-3p & 5'-guggucucuuggccccag-3' \\
\hline 63 & 82 & hsa-miR-6853-3p & 5'-uguucauuggaacccugcgcag-3' \\
\hline 64 & 82 & hsa-miR-1185-2-3p & 5'-auauacagggggagacucucau-3' \\
\hline 65 & 81 & hsa-miR-6736-3p & 5'-ucagcuccucucuacccacag-3' \\
\hline 66 & 81 & hsa-miR-4277 & 5'-gcaguucugagcacaguacac-3' \\
\hline 67 & 81 & hsa-miR-3662 & 5'-gaaaaugaugaguagugacugaug-3' \\
\hline 68 & 81 & hsa-miR-1289 & 5'-uggaguccaggaaucugcauuuu-3' \\
\hline 69 & 81 & hsa-miR-1825 & 5'-uccagugcccuccucucc-3' \\
\hline
\end{tabular}

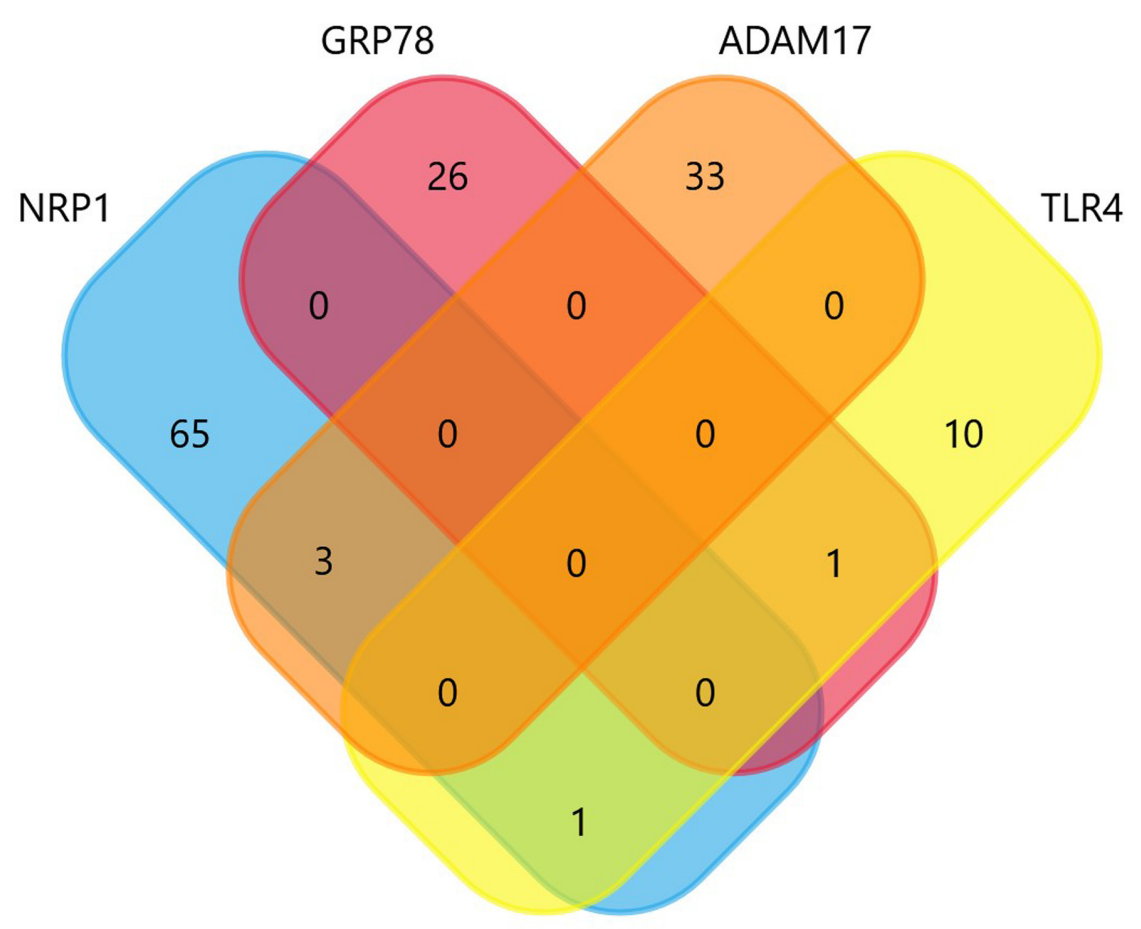

Figure 2. Venn diagram describing shared miRNAs between NRP1, GRP78, ADAM17 and TLR4. NRP1 and ADAM17 share three common miRNAs: hsa-miR-148a-3p, hsa-miR-152-3p and hsa-miR-148b-3p. TLR4 and NRP1 share one common miRNA, hsa-miR-587; whereas TLR4 and GRP78 share hsa-miR-338-5p as a common miRNA.

only one possible interaction identified between a miRNA and CD147 (BSG); Table VIII presents 12 interactions identified between miRNAs and TLR4; and finally, Table IX outlines five possible interactions of these regulatory RNAs with mGluR2.

Further analysis using Venn diagrams (Fig. 2), revealed that there were three common miRNAs amongst NRP1 and
ADAM17, namely hsa-miR-148a-3p, hsa-miR-152-3p and hsa-miR-148b-3p. One common miRNA, i.e., hsa-miR-587, was also shared between TLR4 and NRP1, and another miRNA (hsa-miR-338-5p) between TLR4 and GRP78. Of note, hsa-miR-148a-3p was also shared with ACE2, as previously reported (71). Moreover, hsa-miR-587 was recently identified as a top miRNA regulating ACE2 networks; it was 
Table IV. List of miRNAs that indicate strong binding potential against ADAM metallopeptidase domain 17.

\begin{tabular}{|c|c|c|c|}
\hline Target rank & Target score & miRNA name & miRNA sequence \\
\hline 1 & 99 & hsa-miR-3163 & 5'-uauaaaaugagggcaguaagac-3' \\
\hline 2 & 97 & hsa-miR-548ah-3p & 5'-caaaaacugcaguuacuuuugc-3' \\
\hline 3 & 97 & hsa-miR-548am-3p & 5'-caaaaacugcaguuacuuuugu-3' \\
\hline 4 & 97 & hsa-miR-548x-3p & 5'-uaaaaacugcaauuacuuuc-3' \\
\hline 5 & 97 & hsa-miR-4465 & 5'-cucaaguagucugaccagggga-3' \\
\hline 6 & 97 & hsa-miR-548aj-3p & 5'-uaaaaacugcaauuacuuuua-3' \\
\hline 7 & 96 & hsa-miR-548ae-3p & 5'-caaaaacugcaauuacuuuca-3' \\
\hline 8 & 96 & hsa-miR-548aq-3p & 5'-caaaaacugcaauuacuuuugc-3' \\
\hline 9 & 96 & hsa-miR-1297 & 5'-uucaaguaauucaggug-3' \\
\hline 10 & 96 & hsa-miR-26a-5p & 5'-uucaaguaauccaggauaggcu-3' \\
\hline 11 & 96 & hsa-miR-26b-5p & 5'-uucaaguaauucaggauaggu-3' \\
\hline 12 & 96 & hsa-miR-548j-3p & 5'-caaaaacugcauuacuuuugc-3' \\
\hline 13 & 89 & hsa-miR-507 & 5'-uuuugcaccuuuuggagugaa-3' \\
\hline 14 & 88 & hsa-miR-5697 & 5'-ucaaguaguuucaugauaaagg-3' \\
\hline 15 & 88 & hsa-miR-4762-5p & 5'-ccaaaucuugaucagaagccu-3' \\
\hline 16 & 88 & hsa-miR-4719 & 5'-ucacaaaucuauaauaugcagg-3' \\
\hline 17 & 88 & hsa-miR-5197-5p & 5'-caauggcacaaacucauucuuga-3' \\
\hline 18 & 88 & hsa-miR-4686 & 5'-uaucugcugggcuuucugguguu-3' \\
\hline 19 & 87 & hsa-miR-95-5p & 5'-ucaauaaaugucuguugaauu-3' \\
\hline 20 & 87 & hsa-miR-8064 & 5'-agcacacugagcgagcggac-3' \\
\hline 21 & 86 & hsa-miR-7109-3p & 5'-caagccucuccugcccuuccag-3' \\
\hline 22 & 86 & hsa-miR-513a-5p & 5'-uucacagggaggugucau-3' \\
\hline 23 & 84 & hsa-miR-5195-3p & 5'-auccaguucucugagggggcu-3' \\
\hline 24 & 84 & hsa-miR-557 & 5'-guuugcacgggugggccuugucu-3' \\
\hline 25 & 84 & hsa-miR-145-5p & 5'-guccaguuuucccaggaaucccu-3' \\
\hline 26 & 84 & hsa-miR-12136 & 5'-gaaaaagucauggaggcc-3' \\
\hline 27 & 84 & hsa-miR-6126 & 5'-gugaaggcccggcggaga-3' \\
\hline 28 & 83 & hsa-miR-4799-5p & 5'-aucuaaaugcagcaugccaguc-3' \\
\hline 29 & 83 & hsa-miR-148a-3p & 5'-ucagugcacuacagaacuuugu-3' \\
\hline 30 & 83 & hsa-miR-152-3p & 5'-ucagugcaugacagaacuugg-3' \\
\hline 31 & 83 & hsa-miR-4464 & 5'-aagguuuggauagaugcaaua-3' \\
\hline 32 & 83 & hsa-miR-4748 & 5'-gagguuuggggaggauuugcu-3' \\
\hline 33 & 83 & hsa-miR-605-3p & 5'-agaaggcacuaugagauuuaga-3' \\
\hline 34 & 83 & hsa-miR-148b-3p & 5'-ucagugcaucacagaacuuugu-3' \\
\hline 35 & 83 & hsa-miR-767-5p & 5'-ugcaccaugguugucugagcaug-3' \\
\hline 36 & 81 & hsa-miR-5481 & 5'-aaaaguauuugcggguuuuguc-3' \\
\hline
\end{tabular}

thus concluded that these miRNAs can be used in the personalized diagnosis of patients with COVID-19 (74).

Moreover, making use of the miRNA atlas (ccb-web. cs.uni-saarland.de/tissueatlas), it was evident that the miRNAs listed in Tables I-VIII have a wide distribution in the human body and co-localise with SARS-CoV-2 cell entry mediators (Fig. 3). For example, hsa-miR-152-3p is primarily expressed in the epididymis, colon, artery, oesophagus, thyroid, whereas hsa-miR-338-5p is mostly present in the kidneys, brain and spleen. This co-localisation provides a higher order of complexity on the mechanisms through which these mediators may be regulated by miRNAs. Future studies are required to evaluate their therapeutic or diagnostic potential.

\section{Conclusion}

The pathophysiology of COVID-19 is mainly dependent on the underlying mechanisms that mediate the entry of SARS-CoV-2 into the host cells of the various human tissues/organs. Based on the growing body of evidence regarding these mechanisms, it is evident that there is an array of factors/mediators which play crucial roles in these processes, often in synergistic/complimentary ways. The complexity of these viral-host interactions appears to be further increased when the co-localization of SARS-CoV-2 host cell entry mediators in different human tissues/organs is considered, as well as when considering the potential modulatory effects that several miRNAs can have on 
Table V. List of miRNAs that indicate strong binding potential against hepatitis A virus cellular receptor 1 (HAVCR1, KIM1).

\begin{tabular}{lcll}
\hline Target rank & Target score & \multicolumn{1}{c}{ miRNA name } & \multicolumn{1}{c}{ miRNA sequence } \\
\hline 1 & 90 & hsa-miR-4255 & $5^{\prime}$-caguguucagagaugga-3' \\
2 & 87 & hsa-miR-4738-3p & $5^{\prime}$-'ugaaacuggagcgccuggagga-3' \\
3 & 86 & hsa-miR-3117-3p & $5^{\prime}$ '-auaggacucauauagugccag-3' \\
4 & 85 & hsa-miR-3171 & $5^{\prime}$-agauguauggaaucuguauauauc-3' \\
5 & 83 & hsa-miR-383-5p & $5^{\prime}$-agaucagaaggugauuguggcu-3' \\
\hline
\end{tabular}

Table VI. List of miRNAs that indicate strong binding potential against heat shock protein family A (Hsp70) member 5 (HSPA5 or GRP78).

\begin{tabular}{|c|c|c|c|}
\hline Target rank & Target score & miRNA name & miRNA sequence \\
\hline 1 & 99 & hsa-miR-3121-3p & 5'-uaaauagaguaggcaaaggaca-3' \\
\hline 2 & 97 & hsa-miR-5688 & 5'-uaacaaacaccuguaaaacagc-3' \\
\hline 3 & 96 & hsa-miR-635 & 5'-acuugggcacugaaacaaugucc-3' \\
\hline 4 & 96 & hsa-miR-6774-5p & 5'-acuugggcaggagggacccuguaug-3' \\
\hline 5 & 95 & hsa-miR-495-3p & 5'-aaacaaacauggugcacuucuu-3' \\
\hline 6 & 93 & hsa-miR-338-5p & 5'-aacaauauccuggugcugagug-3' \\
\hline 7 & 91 & hsa-miR-7-2-3p & 5'-caacaaaucccagucuaccuaa-3' \\
\hline 8 & 91 & hsa-miR-7-1-3p & 5'-caacaaaucacagucugccaua-3' \\
\hline 9 & 90 & hsa-miR-30d-3p & 5'-cuuucagucagauguuugcugc-3' \\
\hline 10 & 89 & hsa-miR-199a-5p & 5'-cccaguguucagacuaccuguuc-3' \\
\hline 11 & 89 & hsa-miR-199b-5p & 5'-cccaguguuuagacuaucuguuc-3' \\
\hline 12 & 89 & hsa-miR-4699-3p & 5'-aauuuacucugcaaucuucucc-3' \\
\hline 13 & 88 & hsa-miR-7854-3p & 5'-ugaggugaccgcagaugggaa-3' \\
\hline 14 & 87 & hsa-miR-4650-3p & 5'-agguagaaugaggccugacau-3' \\
\hline 15 & 85 & hsa-miR-222-5p & 5'-cucaguagccaguguagauccu-3' \\
\hline 16 & 84 & hsa-miR-16-1-3p & 5'-ccaguauuaacugugcugcuga-3' \\
\hline 17 & 84 & hsa-miR-30e-3p & 5'-cuuucagucggauguuuacagc-3' \\
\hline 18 & 84 & hsa-miR-148a-5p & 5 '-aaaguucugagacacuccgacu-3' \\
\hline 19 & 84 & hsa-miR-30a-3p & 5'-cuuucagucggauguuugcagc-3' \\
\hline 20 & 84 & hsa-miR-4451 & 5'-ugguagagcugaggaca-3' \\
\hline 21 & 84 & hsa-miR-7162-3p & 5'-ucugagguggaacagcagc-3' \\
\hline 22 & 83 & hsa-miR-6777-3p & 5'-uccacucuccuggcccccag-3' \\
\hline 23 & 82 & hsa-miR-770-5p & 5 '-uccaguaccacgugucagggcca-3' \\
\hline 24 & 82 & hsa-miR-4712-5p & 5'-uccaguacaggucucucauuuc-3' \\
\hline 25 & 81 & hsa-miR-3606-3p & 5'-aaaauuucuuucacuacuuag-3' \\
\hline 26 & 81 & hsa-miR-513c-3p & 5'-uaaauuucaccuuucugagaaga-3' \\
\hline 27 & 81 & hsa-miR-513a-3p & 5'-uaaauuucaccuuucugagaagg-3' \\
\hline
\end{tabular}

Table VII. One miRNA which exhibits strong binding potential against basigin (BSG, CD147).

\begin{tabular}{lccc}
\hline Target rank & Target score & miRNA name & miRNA sequence \\
\hline 1 & 93 & hsa-miR-1252-5p & 5'-agaaggaaauugaauucauuua-3' \\
\hline
\end{tabular}

the local expression of these mediators. Accordingly, intensive research is still warranted in this field in order to precisely characterise these underlying mechanisms, with the ultimate objective of aiding in the development of novel treatments, which will act by blocking the entry of SARS-CoV-2 into susceptible human host cells. 
Table VIII. List of miRNAs that indicate strong binding potential against Toll-like receptor 4.

\begin{tabular}{|c|c|c|c|}
\hline Target rank & Target score & miRNA name & miRNA sequence \\
\hline 1 & 93 & hsa-miR-448 & 5'-uugcauauguaggaugucccau-3' \\
\hline 2 & 91 & hsa-miR-3924 & 5'-auauguauaugugacugcuacu-3' \\
\hline 3 & 89 & hsa-miR-627-3p & 5'-ucuuuucuuugagacucacu-3' \\
\hline 4 & 88 & hsa-miR-338-5p & 5'-aacaauauccuggugcugagug-3' \\
\hline 5 & 88 & hsa-miR-4272 & 5'-cauucaacuagugauugu-3' \\
\hline 6 & 87 & hsa-miR-10397-5p & 5'-uccuugaccugaugcuguaggg-3' \\
\hline 7 & 87 & hsa-miR-5583-3p & 5'-gaauauggguauauuaguuugg-3' \\
\hline 8 & 85 & hsa-miR-4760-3p & 5'-aaauucauguucaaucuaaacc-3' \\
\hline 9 & 85 & hsa-miR-4652-3p & 5'-guucuguuaacccauccccuca-3' \\
\hline 10 & 84 & hsa-miR-1243 & 5'-aacuggaucaauuauaggagug-3' \\
\hline 11 & 81 & hsa-miR-587 & 5'-uuuccauaggugaugagucac-3' \\
\hline 12 & 81 & hsa-miR-4678 & 5'-aagguauuguucagacuuauga-3, \\
\hline
\end{tabular}

Table IX. List of miRNAs that indicate strong binding potential against metabotropic glutamate receptor subtype 2 .

\begin{tabular}{llll}
\hline Target rank & Target score & \multicolumn{1}{c}{ miRNA name } & \multicolumn{1}{c}{ miRNA sequence } \\
\hline 1 & 86 & hsa-miR-4492 & $5^{\prime}$-ggggcugggcgcgcgcc-3' \\
2 & 84 & hsa-miR-555 & $5^{\prime}$-aggguaagcugaaccucugau-3' \\
3 & 83 & hsa-miR-4436b-3p & $5^{\prime}$-cagggcaggaagaaguggacaa-3' \\
4 & 83 & hsa-miR-497-3p & $5^{\prime}$-caaaccacacugugguguuaga-3' \\
5 & 82 & hsa-miR-4498 & $5^{\prime}$-ugggcuggcagggcaagugcug-3' \\
\hline
\end{tabular}

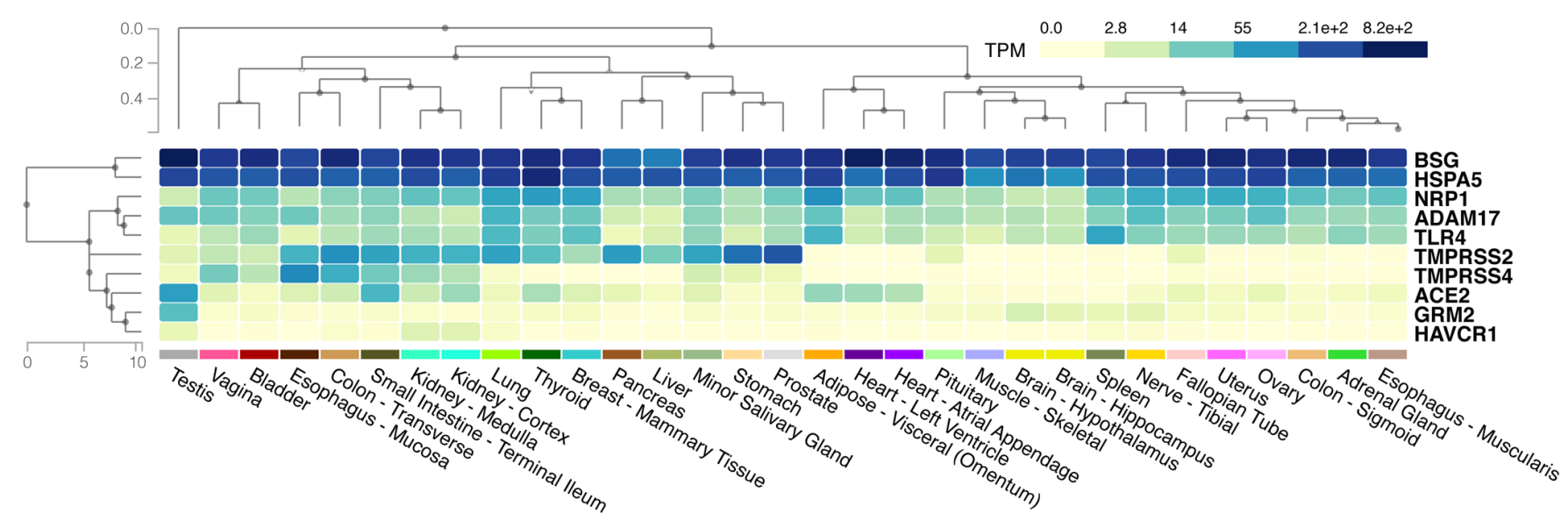

Figure 3. Distribution and co-localisation of all potential cell entry mediators. BSG, basigin (CD147); HSPA5, heat shock protein A5; ADAM17, ADAM metallopeptidase domain 17 (also termed tumour necrosis factor- $\alpha$ convertase); TMPRSS2 and 4, transmembrane protease serine 2 and 4; ACE2, angiotensin converting enzyme 2; GRM2, glutamate receptor subtype 2 (mGluR2); HAVCR1, hepatitis A virus cellular receptor 1 (also termed kidney injury molecule-1, KIM1).

\section{Acknowledgements}

The authors would like to thank Dr Evangelos Kolettas, Laboratory of Biology, School of Medicine, University of Ioannina, and Biomedical Research Division, Institute of Molecular Biology and Biotechnology, Foundation for Research and Technology, Ioannina, Greece, for critically reading and commenting on the manuscript.

\section{Funding}

No funding was received.

\section{Availability of data and materials}

The data used and/or analysed during the current study are available from the corresponding author on reasonable request. 


\section{Authors' contributions}

PK, EK, SS, HSR and IK contributed to the conceptualization, methodology, data curation, investigation, visualization, drafting, editing and reviewing of the manuscript. HSR, DAS and SS contributed to the literature search, and the drafting and critical revision of the manuscript. All authors have read and approved the final manuscript. SS and EK confirm the authenticity of all the raw data.

\section{Ethics approval and consent to participate}

Not applicable.

\section{Patient consent for publication}

Not applicable.

\section{Competing interests}

DAS is the Editor-in-Chief for the journal, but had no personal involvement in the reviewing process, or any influence in terms of adjudicating on the final decision, for this article. The other authors declare that they have no competing interests.

\section{References}

1. Osuchowski MF, Winkler MS, Skirecki T, Cajander S, Shankar-Hari M, Lachmann G, Monneret G, Venet F, Bauer M, Brunkhorst FM, et al: The COVID-19 puzzle: Deciphering pathophysiology and phenotypes of a new disease entity. Lancet Respir Med 9: 622-642, 2021.

2. Singh S, Pandey R, Tomar S, Varshney R, Sharma D and Gangenahalli G: A brief molecular insight of COVID-19: Epidemiology, clinical manifestation, molecular mechanism, cellular tropism and immuno-pathogenesis. Mol Cell Biochem 476: 3987-4002, 2021.

3. Murgolo N, Therien AG, Howell B, Klein D, Koeplinger K, Lieberman LA, Adam GC, Flynn J, McKenna $P$, Swaminathan $\mathrm{G}$, et al: SARS-CoV-2 tropism, entry, replication, and propagation: Considerations for drug discovery and development. PLoS Pathog 17: e1009225, 2021.

4. Gao S and Zhang L: ACE2 partially dictates the host range and tropism of SARS-CoV-2. Comput Struct Biotechnol J 18: 4040-4047, 2020.

5. Hoffmann M, Kleine-Weber H, Schroeder S, Krüger N, Herrler T, Erichsen S, Schiergens TS, Herrler G, Wu NH, Nitsche A, et al SARS-CoV-2 cell entry depends on ACE2 and TMPRSS2 and is blocked by a clinically proven protease inhibitor. Cell 181 : 271-280.e8, 2020.

6. Cai L, Guo X, Cao Y, Ying P, Hong L, Zhang Y, Yi G and Fu M: Determining available strategies for prevention and therapy: Exploring COVID-19 from the perspective of ACE2 (Review). Int J Mol Med 47: 43, 2021.

7. Glowacka I, Bertram S, Müller MA, Allen P, Soilleux E, Pfefferle S, Steffen I, Tsegaye TS, He Y, Gnirss K, et al: Evidence that TMPRSS2 activates the severe acute respiratory syndrome coronavirus spike protein for membrane fusion and reduces viral control by the humoral immune response. J Virol 85: 4122-4134, 2011.

8. Piva F, Sabanovic B, Cecati M and Giulietti M: Expression and Co-expression analyses of TMPRSS2, a key element in COVID-19. Eur J Clin Microbiol Infect Dis 40: 451-455, 2021

9. Hou Y, Zhao J, Martin W, Kallianpur A, Chung MK, Jehi L, Sharifi N, Erzurum S, Eng C and Cheng F: New insights into genetic susceptibility of COVID-19: An ACE2 and TMPRSS2 polymorphism analysis. BMC Med 18: 216, 2020.

10. Zipeto D, Palmeira JDF, Argañaraz GA and Argañaraz ER: ACE2/ADAM17/TMPRSS2 interplay may be the main risk factor for COVID-19. Front Immunol 11: 576745, 2020.
11. Shang J, Wan Y, Luo C, Ye G, Geng Q, Auerbach A and Li F: Cell entry mechanisms of SARS-CoV-2. Proc Natl Acad Sci USA 117: 11727-11734, 2020

12. Magrone T, Magrone M and Jirillo E: Focus on receptors for coronaviruses with special reference to Angiotensin-converting Enzyme 2 as a potential drug Target-A perspective. Endocr Metab Immune Disord Drug Targets 20: 807-811, 2020.

13. Jia H, Neptune E and Cui H: Targeting ACE2 for COVID-19 therapy: Opportunities and challenges. Am J Respir Cell Mol Biol 64: 416-425, 2021.

14. Imai Y, Kuba K, Rao S, Huan Y, Guo F, Guan B, Yang P, Sarao R, Wada T, Leong-Poi H, et al: Angiotensin-converting enzyme 2 protects from severe acute lung failure. Nature 436: 112-116, 2005.

15. Schuler BA, Habermann AC, Plosa EJ, Taylor CJ, Jetter C, Negretti NM, Kapp ME, Benjamin JT, Gulleman P, Nichols DS, et al: Age-determined expression of priming protease TMPRSS2 and localization of SARS-CoV-2 in lung epithelium. J Clin Invest 131: e140766, 2021.

16. Musso N, Falzone L, Stracquadanio S, Bongiorno D, Salerno M, Esposito M, Sessa F, Libra M, Stefani S and Pomara C: Post-mortem detection of SARS-CoV-2 RNA in Long-buried lung samples. Diagnostics (Basel) 11: 1158, 2021.

17. Deinhardt-Emmer S, Wittschieber D, Sanft J, Kleemann S, Elschner S, Haupt KF, Vau V, Häring C, Rödel J, Henke A, et al: Early postmortem mapping of SARS-CoV-2 RNA in patients with COVID-19 and the correlation with tissue damage. Elife 10: e60361, 2021

18. Yao XH, Luo T, Shi Y, He ZC, Tang R, Zhang PP, Cai J, Zhou XD, Jiang DP, Fei XC, et al: A cohort autopsy study defines COVID-19 systemic pathogenesis. Cell Res 31: 836-846, 2021.

19. Zang R, Gomez Castro MF, McCune BT, Zeng Q, Rothlauf PW, Sonnek NM, Liu Z, Brulois KF, Wang X, Greenberg HB, et al: TMPRSS2 and TMPRSS4 promote SARS-CoV-2 infection of human small intestinal enterocytes. Sci Immunol 5: eabc3582, 2020.

20. Kyrou I, Randeva HS, Spandidos DA and Karteris E: Not only ACE2-the quest for additional host cell mediators of SARS-CoV-2 infection: Neuropilin-1 (NRP1) as a novel SARS-CoV-2 host cell entry mediator implicated in COVID-19. Signal Transduct Target Ther 6: 21, 2021.

21. Katopodis P, Kerslake R, Davies J, Randeva HS, Chatha K, Hall M, Spandidos DA, Anikin V, Polychronis A, Robertus JL, et al: COVID-19 and SARS-CoV-2 host cell entry mediators: Expression profiling of TMRSS4 in health and disease. Int J Mol Med 47: 64, 2021.

22. Cuervo NZ and Grandvaux N: ACE2: Evidence of role as entry receptor for SARS-CoV-2 and implications in comorbidities. Elife 9: e61390, 2020.

23. Davies J, Randeva HS, Chatha K, Hall M, Spandidos DA, Karteris E and Kyrou I: Neuropilin-1 as a new potential SARS-CoV-2 infection mediator implicated in the neurologic features and central nervous system involvement of COVID-19. Mol Med Rep 22: 4221-4226, 2020.

24. Daly JL, Simonetti B, Antón-Plágaro C, Williamson MK, Shoemark DK, Simón-Gracia L, Klein K, Bauer M, Hollandi R, Greber UF, et al: Neuropilin-1 is a host factor for SARS-CoV-2 infection. Science 370: 861-865, 2020.

25. Cantuti-Castelvetri L, Ojha R, Pedro LD, Djannatian M, Franz J, Kuivanen S, van der Meer F, Kallio K, Kaya T, Anastasina M, et al: Neuropilin-1 facilitates SARS-CoV-2 cell entry and infectivity. Science 370: 856-860, 2020.

26. Kielian M: Enhancing host cell infection by SARS-CoV-2 . Science 370: 765-766, 2020.

27. Cantuti-Castelvetri L, Ojha R, Pedro LD, Djannatian M, Franz J, Kuivanen S, Kallio K, Kaya T, Anastasina M, Smura T, et al: Neuropilin-1 facilitates SARS-CoV-2 cell entry and provides a possible pathway into the central nervous system. bioRxiv: Jul 15 , 2020 (Epub ahead of print). doi: 10.1101/2020.06.07.137802.

28. Elfiky AA: SARS-CoV-2 Spike-heat shock protein A5 (GRP78) recognition may be related to the immersed human coronaviruses. Front Pharmacol 11: 577467, 2020.

29. Ibrahim IM, Abdelmalek DH, Elshahat ME and Elfiky AA: COVID-19 spike-host cell receptor GRP78 binding site prediction. J Infect 80: 554-562, 2020.

30. Wang K, Chen W, Zhang Z, Deng Y, Lian JQ, Du P, Wei D, Zhang Y, Sun XX, Gong L, et al: CD147-spike protein is a novel route for SARS-CoV-2 infection to host cells. Signal Transduct Target Ther 5: 283, 2020.

31. Shilts J, Crozier TWM, Greenwood EJD, Lehner PJ and Wright GJ: No evidence for basigin/CD147 as a direct SARS-CoV-2 spike binding receptor. Sci Rep 11: 413, 2021. 
32. Minami T, Iwata Y and Wada T: Renal complications in coronavirus disease 2019: A systematic review. Inflamm Regen 40: $31,2020$.

33. Yang C, Zhang Y, Zeng X, Chen H, Chen Y, Yang D, Shen Z, Wang X, Liu X, Xiong M, et al: Kidney injury molecule-1 is a potential receptor for SARS-CoV-2. J Mol Cell Biol 13: 185-196, 2021.

34. Wan $\mathrm{C}$ and Zhang C: Kidney injury molecule-1: A novel entry factor for SARS-CoV-2. J Mol Cell Biol 13: 159-160, 2021.

35. Bu Z, Wang J, Yang G, Wang X, Wen Z, Shuai L, Luo J, Wang C, Sun Z, Liu R, et al: Metabotropic glutamate receptor subtype 2 is a receptor of SARS-CoV-2. Res Sq: April 21, 2021 (Epub ahead of print). doi: 10.21203/rs.3.rs-301270/v1.

36. Cui C, Huang C, Zhou W, Ji X, Zhang F, Wang L, Zhou Y and Cui Q: AGTR2, one possible novel key gene for the entry of SARS-CoV-2 into human cells. IEEE/ACM Trans Comput Biol Bioinforma 18: 1230-1233, 2021.

37. Zhang Q, Chen CZ, Swaroop M, Xu M, Wang L, Lee J, Wang AQ, Pradhan M, Hagen N, Chen L, et al: Heparan sulfate assists SARS-CoV-2 in cell entry and can be targeted by approved drugs in vitro. Cell Discov 6: 80, 2020.

38. Lambert DW, Yarski M, Warner FJ, Thornhill P, Parkin ET, Smith AI, Hooper NM and Turner AJ: Tumor necrosis factor-alpha convertase (ADAM17) mediates regulated ectodomain shedding of the severe-acute respiratory syndrome-coronavirus (SARS-CoV) receptor, angiotensin-converting enzyme-2 (ACE2). J Biol Chem 280: 30113-30119, 2005.

39. Palau V, Riera M and Soler MJ: ADAM17 inhibition may exert a protective effect on COVID-19. Nephrol Dial Transplant 35: 1071-1072, 2020

40. Heurich A, Hofmann-Winkler H, Gierer S, Liepold T, Jahn O and Pöhlmann S: TMPRSS2 and ADAM17 cleave ACE2 differentially and only proteolysis by TMPRSS 2 augments entry driven by the severe acute respiratory syndrome coronavirus spike protein. J Virol 88: 1293-1307, 2014.

41. Kumar J, Murugaiah V, Sotiriadis G, Kaur A, Jeyaneethi J, Sturniolo I, Alhamlan FS, Chatterjee J, Hall M, Kishore U and Karteris E: Surfactant Protein D as a potential biomarker and therapeutic target in ovarian cancer. Front Oncol 9: 542, 2019.

42. Hsieh MH, Beirag N, Murugaiah V, Chou YC, Kuo WS, Kao HF Madan T, Kishore U and Wang JY: Human surfactant Protein D binds spike protein and acts as an entry inhibitor of SARS-CoV-2 pseudotyped viral particles. Front Immunol 12: 641360, 2021.

43. Madan T, Biswas B, Varghese PM, Subedi R, Pandit H, Idicula-Thomas S, Kundu I, Rooge S, Agarwal R, Tripathi DM, etal: A recombinant fragment of human surfactant Protein D binds spike protein and inhibits infectivity and replication of SARS-CoV-2 in clinical samples. Am J Respir Cell Mol Biol 65: 41-53, 2021.

44. Tong M, Xiong Y, Zhu C, Xu H, Zheng Q, Jiang Y, Zou L, Xiao X, Chen F, Yan X, et al: Serum surfactant protein D in COVID-19 is elevated and correlated with disease severity. BMC Infect Dis 21: 737,2021

45. Aboudounya MM and Heads RJ: COVID-19 and toll-like receptor 4 (TLR4): SARS-CoV-2 may bind and activate TLR4 to increase ACE2 expression, facilitating entry and causing hyperinflammation. Mediators Inflamm 2021: 8874339, 2021.

46. Gadanec LK, McSweeney KR, Qaradakhi T, Ali B, Zulli A and Apostolopoulos V: Can SARS-CoV-2 virus use multiple receptors to enter host cells? Int J Mol Sci 22: 992, 2021.

47. Zhao Y, Kuang M, Li J, Zhu L, Jia Z, Guo X, Hu Y, Kong J, Yin $\mathrm{H}$, Wang $\mathrm{X}$ and You F: SARS-CoV-2 spike protein interacts with and activates TLR41. Cell Res 31: 818-820, 2021.

48. Zamorano Cuervo N and Grandvaux N: ACE2: Evidence of role as entry receptor for SARS-CoV-2 and implications in comorbidities. Elife 9: e61390, 2020

49. Lan J, Ge J, Yu J, Shan S, Zhou H, Fan S, Zhang Q, Shi X, Wang Q, Zhang L and Wang X: Structure of the SARS-CoV-2 spike receptor-binding domain bound to the ACE2 receptor. Nature 581: 215-220, 2020.

50. Shrimp JH, Kales SC, Sanderson PE, Simeonov A, Shen M and Hall MD: An enzymatic TMPRSS2 assay for assessment of clinical candidates and discovery of inhibitors as potential treatment of COVID-19. ACS Pharmacol Transl Sci 3: 997-1007, 2020.

51. Lee JJ, Kopetz S, Vilar E, Shen JP, Chen K and Maitra A: Relative abundance of SARS-CoV-2 entry genes in the enterocytes of the lower gastrointestinal tract. Genes (Basel) 11: 645, 2020.

52. Choudhury A and Mukherjee S: In silico studies on the comparative characterization of the interactions of SARS-CoV-2 spike glycoprotein with ACE-2 receptor homologs and human TLRs. J Med Virol 92: 2105-2113, 2020.
53. Dexheimer PJ and Cochella L: MicroRNAs: From mechanism to organism. Front Cell Dev Biol 8: 409, 2020

54. Peng Y and Croce CM: The role of MicroRNAs in human cancer. Signal Transduct Target Ther 1: 15004, 2016.

55. Chen K and Rajewsky N: The evolution of gene regulation by transcription factors and microRNAs. Nat Rev Genet 8: 93-103, 2007.

56. Correia de Sousa M, Gjorgjieva M, Dolicka D, Sobolewski C and Foti M: Deciphering miRNAs' Action through miRNA Editing. Int J Mol Sci 20: 6249, 2019.

57. Cai Y, Yu X, Hu S and Yu J: A brief review on the mechanisms of miRNA regulation. Genomics Proteomics Bioinformatics 7: $147-154,2009$

58. Liu Z, Wang J, Ge Y, Xu Y, Guo M, Mi K, Xu R, Pei Y, Zhang Q, Luan $\mathrm{X}$, et al: SARS-CoV-2 encoded microRNAs are involved in the process of virus infection and host immune response. J Biomed Res 35: 216-227, 2021.

59. Chauhan N, Jaggi M, Chauhan SC and Yallapu MM: COVID-19: Fighting the invisible enemy with microRNAs. Expert Rev Anti Infect Ther 19: 137-145, 2021.

60. Fani M,Zandi M,Ebrahimi S, Soltani S and Abbasi S: The role of miRNAs in COVID-19 disease. Future Virol 16: 301-306, 2021.

61. Bugnon LA, Raad J, Merino GA, Yones C, Ariel F, Milone DH and Stegmayer G: Deep Learning for the discovery of new pre-miRNAs: Helping the fight against COVID-19. Mach Learn with Appl 6: 100150, 2021.

62. Abedi F, Rezaee R, Hayes AW, Nasiripour S and Karimi G: MicroRNAs and SARS-CoV-2 life cycle, pathogenesis, and mutations: Biomarkers or therapeutic agents? Cell Cycle 20: 143-153, 2021.

63. Haddad $\mathrm{H}$ and $\mathrm{Al}-\mathrm{Zyoud} \mathrm{W}$ : miRNA target prediction might explain the reduced transmission of SARS-CoV-2 in Jordan, Middle East. Noncoding RNA Res 5: 135-143, 2020.

64. Sardar R, Satish D, Birla S and Gupta D: Integrative analyses of SARS-CoV-2 genomes from different geographical locations reveal unique features potentially consequential to host-virus interaction, pathogenesis and clues for novel therapies. Heliyon 6: e04658, 2020.

65. Matarese A, Gambardella J, Sardu C and Santulli G: miR-98 regulates TMPRSS2 expression in human endothelial cells: Key implications for COVID-19. Biomedicines 8: 462, 2020.

66. Nersisyan S, Shkurnikov M, Turchinovich A, Knyazev E and Tonevitsky A: Integrative analysis of miRNA and mRNA sequencing data reveals potential regulatory mechanisms of ACE2 and TMPRSS2. PLoS One 15: e0235987, 2020.

67. Lu D, Chatterjee S, Xiao K, Riedel I, Wang Y, Foo R, Bär C and Thum T: MicroRNAs targeting the SARS-CoV-2 entry receptor ACE2 in cardiomyocytes. J Mol Cell Cardiol 148: 46-49, 2020.

68. Khan MA, Sany MRU, Islam MS and Islam ABMMK: Epigenetic regulator miRNA pattern differences among SARS-CoV, SARS-CoV-2, and SARS-CoV-2 World-Wide isolates delineated the mystery behind the epic pathogenicity and distinct clinical characteristics of pandemic COVID-19. Front Genet 11: 765, 2020.

69. Paul S, Bravo Vázquez LA, Reyes-Pérez PR, Estrada-Meza C, Aponte Alburquerque RA, Pathak S, Banerjee A, Bandyopadhyay A, Chakraborty S and Srivastava A: The role of microRNAs in solving COVID-19 puzzle from infection to therapeutics: A mini-review. Virus Res 308: 198631, 2021.

70. Dash S, Dash C and Pandhare J: Therapeutic significance of microRNA-mediated regulation of PARP-1 in SARS-CoV-2 infection. Noncoding RNA 7: 60, 2021.

71. Mukhopadhyay D and Mussa BM: Identification of novel hypothalamic MicroRNAs as promising therapeutics for SARS-CoV-2 by regulating ACE2 and TMPRSS2 expression: An in silico analysis. Brain Sci 10: 666, 2020.

72. Wang X: miRDB: A microRNA target prediction and functional annotation database with a wiki interface. RNA 14: 1012-1017, 2008.

73. Wong $\mathrm{N}$ and Wang X: miRDB: An online resource for microRNA target prediction and functional annotations. Nucleic Acids Res 43: D146-D152, 2015.

74. Wicik Z, Eyileten C, Jakubik D, Simões SN, Martins DC Jr, Pavão R, Siller-Matula JM and Postula M: ACE2 interaction networks in COVID-19: A physiological framework for prediction of outcome in patients with cardiovascular risk factors. J Clin Med 9: 3743, 2020

This work is licensed under a Creative Commons Attribution-NonCommercial-NoDerivatives 4.0 International (CC BY-NC-ND 4.0) License. 